\title{
Widely tunable picosecond optical parametric oscillator using highly nonlinear fiber
}

\author{
Yue Zhou, * Kim K. Y. Cheung, Sigang Yang, P. C. Chui, and Kenneth K. Y. Wong \\ Department of Electrical and Electronic Engineering, The University of Hong Kong, \\ Pokfulam Road, Hong Kong \\ *Corresponding author: yzhou@eee.hku.hk
}

Received December 1, 2008; revised February 5, 2009; accepted February 18, 2009; posted February 23, 2009 (Doc. ID 104759); published March 20, 2009

\begin{abstract}
We demonstrated a fully fiber-integrated widely tunable picosecond optical parametric oscillator based on highly nonlinear fiber. The ring cavity with a $50 \mathrm{~m}$ highly nonlinear fiber was synchronously pumped with a picosecond mode-locked fiber laser. The tuning range was from 1413 to $1543 \mathrm{~nm}$ and from 1573 to $1695 \mathrm{~nm}$, which was as wide as $250 \mathrm{~nm}$. A high-quality pulse was generated with a pulse width narrower than that of the pump. (C) 2009 Optical Society of America

OCIS codes: $060.2320,060.4370,190.4380$.
\end{abstract}

Picosecond optical pulse sources have been playing an important role in ultrafast processes and optical communications. Today there is a growing demand for short pulse generation in nonconventional wavelength bands. However, a high-quality picosecond laser is generally not available at some nonconventional wavelengths due to the lack of efficient gain medium. Thanks to the ultrafast response, wide-gain bandwidth [1], high gain [2], and large detune from the pump [1] of the fiber optical parametric amplifier (FOPA), efficient short pulse generation is possible at nonconventional wavelengths by using an optical parametric oscillator (OPO) configuration.

Conventionally, an OPO based on the $\chi^{(2)}$ nonlinear effect of crystals is utilized for parametric oscillation [3]. This technique has a wide range of applications in optical research and development. However, this approach requires a dedicated alignment for optimal operation. Fiber optical parametric oscillators (FOPOs), which are based on the $\chi^{(3)}$ nonlinear effect of optical fiber, eliminate the need for alignment and allow further integration with fiber components [4]. Using the FOPO configuration, $\mathrm{CW}$ [5-7] and nanosecond [8] pulse generators have been demonstrated with a wide wavelength tuning range. For picosecond and femtosecond pulses, since the walk-off between the pump and the signal is quite large, it is required to use short fiber, especially microstructure fiber (MF) based FOPOs, which have a tuning range mostly in the visible regime [9-11]. However, most of the telecommunications applications require sources in the spectral region near $1550 \mathrm{~nm}$. Some other MF based FOPOs [12], dispersion-shifted fiber (DSF) based FOPOs [13], and highly nonlinear fiber (HNLF) based FOPOs [14,15] were designed to operated in this regime, but usually with limited tuning range. Thus, to build a widely tunable FOPO operated around $1550 \mathrm{~nm}$ is highly desirable.

In this Letter, we demonstrated a fully fiberintegrated widely tunable picosecond optical parametric oscillator based on HNLF. The tuning range was as wide as $250 \mathrm{~nm}$ around $1550 \mathrm{~nm}$. The totally fiber-integrated nature of this source allows complete self-alignment and further integration to other sys- tems. This scheme may be useful in generating a short pulse for potential ultrafast communication in nonconventional wavelength bands.

When a highly nonlinear DSF (HNL-DSF) is deployed as the gain medium inside a cavity, the amplified spontaneous emission (ASE) noise in either of the sidebands will be amplified and then fed back to the HNL-DSF as a seed. After multiple paths there will be significant signal and idler components at the output.

A phase-matching technique is required to generate signal and idler with substantial power. The phase-matching condition is

$$
\Delta \beta+2 \gamma P=0,
$$

where $\gamma$ is the fiber nonlinear coefficient and $P$ is the incident pump power. $\Delta \beta$ is the total phase mismatch, which can be approximated by the following equation [1]:

$$
\Delta \beta=\beta^{(2)}(\Delta \omega)^{2}+\beta^{(4)}(\Delta \omega)^{4} / 12,
$$

where $\Delta \omega$ is the frequency detune of the signal (and therefore idler) from the pump; $\beta^{(m)}$ is the $m$ th order derivative of the propagation constant.

The shape of the gain spectrum is determined by the fiber dispersion profile. When $\beta^{(2)}<0$ and $\beta^{(4)}$ $<0$, it will operate as a conventional FOPA, which has a continuous gain spectrum around the pump. One the other hand, when $\beta^{(2)}>0$ and $\beta^{(4)}<0$, idler and signal will be generated with frequency detune much larger than the bandwidth of the conventional FOPA. For a given fiber, we can vary $\beta^{(2)}$ over a large range by tuning the pump wavelength, thus making the widely tunable FOPO feasible.

The experimental setup of a widely tunable picosecond pulse generator is shown in Fig. 1. The pump was generated by a picosecond mode-locked fiber laser (MLFL), which generated a short pulse with repetition rate of $10 \mathrm{GHz}$ and a pulse width of $10 \mathrm{ps}$. The output from the pulsed laser was intensitymodulated by a $156.2 \mathrm{MHz}$ electrical pulse with duty ratio of 1/64 to increase the peak power of the pump, and broadened to $15 \mathrm{ps}$ by a variable-bandwidth tun- 


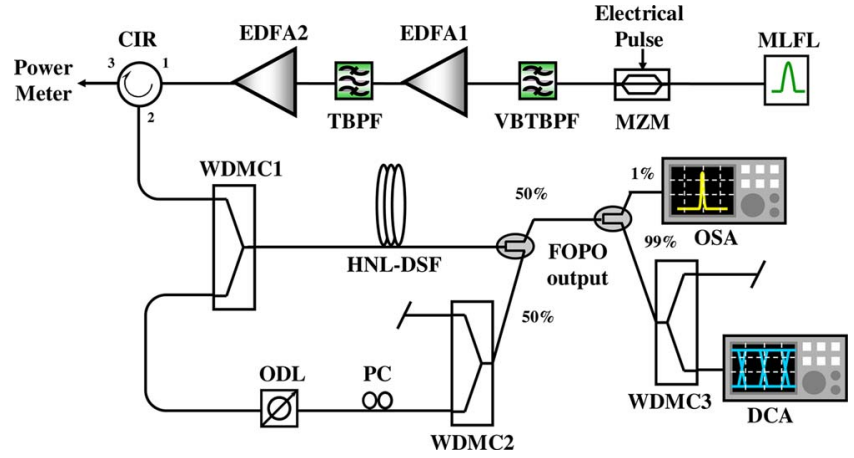

Fig. 1. (Color online) Experimental setup of the FOPO. EDFA, erbium-doped fiber amplifier; TBPF, tunable bandpass filter; OSA, optical spectrum analyzer; MZM, MachZehnder modulator; DCA, digital communication analyzer; PC, polarization controller; ODL, optical delay line; CIR, circulator.

able bandpass filter (VBTBPF), which has a $3 \mathrm{~dB}$ bandwidth of $0.3 \mathrm{~nm}$ to produce a pump pulse with a duty ratio of $0.23 \%$ and a repetition rate of 156.2 $\mathrm{MHz}$. The motivation of increasing the pulse width from 10 to $15 \mathrm{ps}$ was to increase the interaction length of the pump and the signal to obtain a higher gain. The pump was then amplified by erbium-doped fiber amplifiers (EDFAs), EDFA1 and EDFA2, and filtered by a tunable bandpass filter (TBPF) with a $1 \mathrm{~nm}$ bandwidth to produce a highpower, low-noise pump. The average power of the pump was measured to be $20 \mathrm{~mW}$ after the amplifier. If we take duty ratio into consideration, the peak pump power was estimated to be $8.7 \mathrm{~W}$. The pump then passed through a circulator, and the reflected power was observed using a power monitor. Since the linewidth of the pump pulse was larger than the bandwidth of the stimulated Brillouin scattering (SBS) gain, the SBS was low and therefore the phase modulator used in [7] was not required to suppress the SBS. It was then coupled into the cavity for parametric amplification through the wavelength-division multiplexing coupler (WDMC1), which had a cutoff wavelength of $1565 \mathrm{~nm}$. Note that the cavity length was about $60 \mathrm{~m}$, including a $50 \mathrm{~m}$ long HNL-DSF as the gain medium, which had nonlinear coefficient of $14 \mathrm{~W}^{-1} \mathrm{~km}^{-1}$, zero-dispersion wavelength (ZDW) of $1554.7 \mathrm{~nm}$, and dispersion slope of $0.035\left(\mathrm{ps} / \mathrm{nm}^{2}\right) / \mathrm{km}$. A $50 / 50$ coupler in the cavity provided $50 \%$ feedback and $50 \%$ output. The feedback branch was filtered by the WDMC2 with a cutoff wavelength of $1565 \mathrm{~nm}$, so that only the signal (Stokes wave) returned to the HNL-DSF through WDMC1. As a result, the FOPO was only singly resonant with the signal. The polarization controller (PC) in the cavity was used to align the state of polarization (SOP) with that of the pump, while the optical line delay (ODL) in the cavity was used to adjust the cavity length, thus the round-trip time of the signal matched the pump repetition rate, so as to synchronize the signal with the pump.

After one round trip, the walk-off of the pump and the signal along the fiber was approximately 8 ps for a $100 \mathrm{~nm}$ separation, which was comparable to the pump pulse width. However, by changing the cavity length, the signal could be synchronized with a new pump pulse after one round trip, thus the walk-off between the pump and the signal could be compensated and would not aggregate. The round-trip loss of the cavity was measured to be $7.1 \mathrm{~dB}$. The singlepass signal peak gain of the FOPO in the case of perfect phase matching can be calculated using [8]

$$
G_{\mathrm{dB}} \approx 10 \log _{10}\left[\frac{1}{4} \exp (2 \gamma P L)\right],
$$

where $L$ is the fiber length, $P$ is the peak power of the pump, and $\gamma$ is the nonlinear coefficient. The gain was calculated to be $\sim 46 \mathrm{~dB}$. The actual gain was perceived to be lower than this value, since not only the walk-off between the signal and the pump would lower the gain: ZDW fluctuations and random birefringence along the HNL-DSF would also lower the gain [1]. However, the gain can still easily exceed the cavity loss. Stable mode-locked oscillations thus occurred.

The FOPO output spectrum was monitored by an OSA through a 1/99 coupler. WDMC3 was used to filter out the desired signal or idler. The waveform of the signal (idler) was recorded using a digital communication analyzer (DCA).

Figure 2 shows the optical spectra measured at the FOPO output port. The pump wavelength was tuned from 1559 to $1544 \mathrm{~nm}$ and the pump power was slightly adjusted to maintain almost the same peak and narrow linewidth for all spectra. When the ODL was tuned slightly at the same time, the achievable output tuning range was from 1413.5 to $1543.7 \mathrm{~nm}$ and from 1573.4 to $1695.8 \mathrm{~nm}$, which was over $250 \mathrm{~nm}$. The tuning range is larger than those CW [5-7] and picosecond FOPOs [12-15] reported. The widest separation from the pump was $151.8 \mathrm{~nm}$. The smaller peaks that occurred when the pump wavelength was tuned to above $1554 \mathrm{~nm}$ were high-order four-wave mixing (FWM) components. The narrow linewidth $(\sim 2 \mathrm{~nm})$ of the output signal or idler that requires no additional bandpass filters inside the cavity to select the wavelengths is a highly desirable

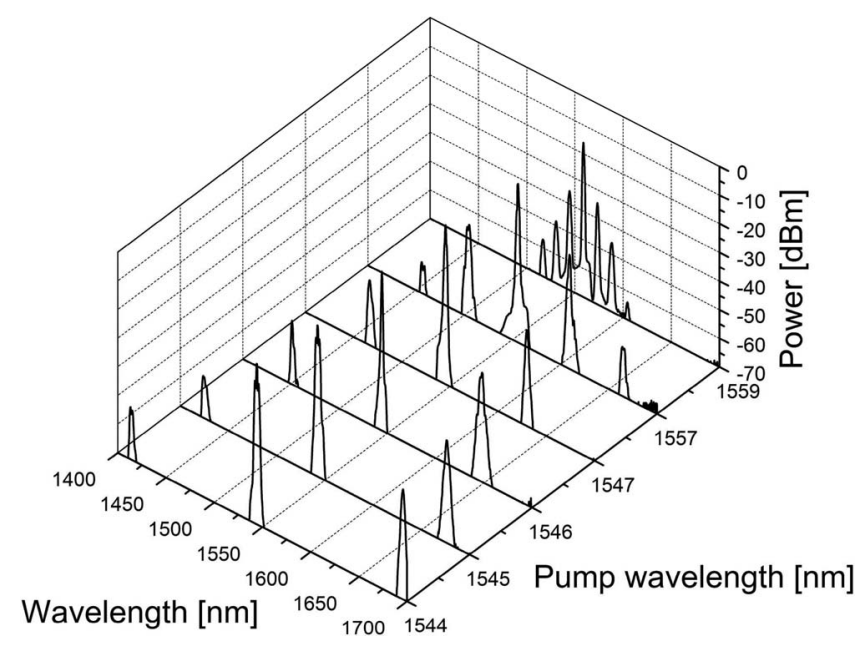

Fig. 2. Optical spectra measured at FOPO output using OSA. 


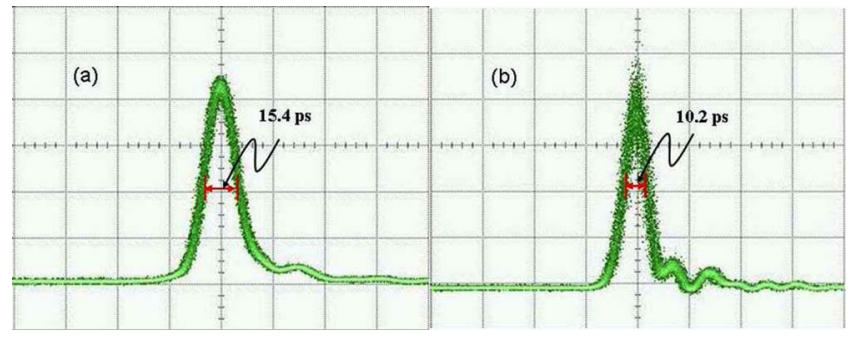

Fig. 3. (Color online) (a) Output pulse from the pump source and (b) signal pulse observed at the FOPO output. The horizontal scale is $20 \mathrm{ps} / \mathrm{div}$.

feature for this configuration, as the availability of the filter at certain wavelengths may limit the tuning range of the FOPO [7].

The long wavelengths's amplitudes that were larger than those of the short wavelengths when their separations to the pump wavelength were large may be due to the Raman gain. Further tuning was limited by the walk-off between the pump and the signal, which increased with their wavelength separation and thereby decreased their interaction length.

Figure 3 shows the waveforms of the input pump pulse at $1544 \mathrm{~nm}$ and output signal pulse at $1695 \mathrm{~nm}$ measured from the DCA. It can be observed that the pulse width of the signal was narrower than that of the pump because of the pulse compression effect [16]. The compression ratio was less than that predicted because of the walk-off between the pump and the signal. The signal-to-noise ratio (SNR) of the signal measured from the DCA was $17 \mathrm{~dB}$. The noise at the mark level was inherited from the ASE noise of the EDFAs. The actual signal pulse width was believed to be shorter than the measured value as the high amplitude ringing at the trailing edge suggested that the measurement was limited by the photodetector electrical bandwidth of about $32 \mathrm{GHz}$.

Figure 4 shows the average output power of the signal near $1695 \mathrm{~nm}$ from the FOPO output port ver-

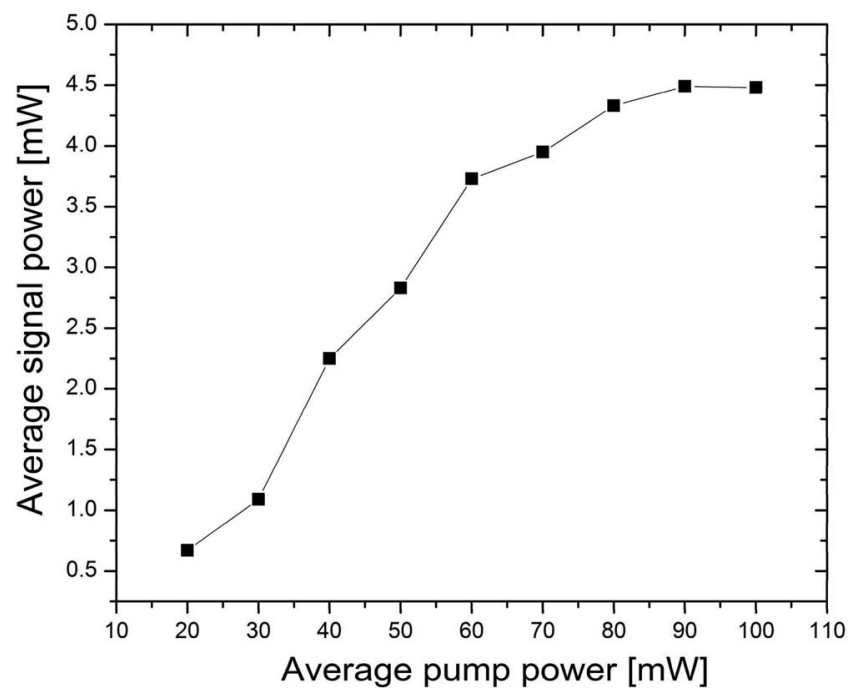

Fig. 4. Average output power of the signal from FOPO as the function of pump power. sus the average power of the pump of wavelength at $1544 \mathrm{~nm}$. Gain saturation began as the average pump power exceeded $80 \mathrm{~mW}$, indicating that further increasing the pump power would not generate a much higher signal output power as discussed in [6]. The conversion efficiency was from $3.35 \%$ to $6.21 \%$, which was higher than those picosecond FOPOs reported in $[12,13]$. The conversion efficiency was perceived to be limited by the walk-off between the signal and the pump.

In conclusion, an all-fiber widely tunable picosecond optical parametric oscillator based on highly nonlinear fiber was demonstrated. The output was continuously tunable from 1413 to $1543 \mathrm{~nm}$ and from 1573 to $1695 \mathrm{~nm}$, when the pump wavelength was tuned between 1559 and $1544 \mathrm{~nm}$. A high-quality pulse was generated with a pulse width narrower than that of the pump. This scheme could be found useful in the generation short pulses for ultrafast communication in nonconventional wavelength bands.

The work described in this Letter was partially supported by grants from the Research Grants Council of the Hong Kong Special Administrative Region, China (projects HKU 7172/07E and HKU 7179/08E). The authors also acknowledge Sumitomo Electric Industries for providing the HNL-DSF and Alnair Laboratories for providing the VBTBPF.

\section{References}

1. M. E. Marhic, K. K.-Y. Wong, and L. G. Kazovsky, IEEE J. Sel. Top. Quantum Electron. 10, 1133 (2004).

2. T. Torounidis, P. A. Andrekson, and B.-E. Olsson, IEEE Photon. Technol. Lett. 18, 1194 (2006).

3. M. H. Dunn and M. Ebrahimzadeh, Science 286, 1513 (1999).

4. J. E. Sharping, J. Lightwave Technol. 26, 2184 (2008).

5. M. E. Marhic, K. K. Y. Wong, L. G. Kazovsky, and T. E. Tsai, Opt. Lett. 27, 1439 (2002).

6. C. de Matos, J. Taylor, and K. Hansen, Opt. Lett. 29, 983 (2004).

7. T. Torounidis and P. Andrekson, IEEE Photon. Technol. Lett. 19, 650 (2007).

8. G. K. L. Wong, S. G. Murdoch, R. Leonhardt, J. D. Harvey, and V. Marie, Opt. Express 15, 2947 (2007).

9. Y. Deng, Q. Lin, F. Lu, G. Agrawal, and W. Knox, Opt. Lett. 30, 1234 (2005).

10. Y. Q. Xu, S. G. Murdoch, R. Leonhardt, and J. D. Harvey, Opt. Lett. 33, 1351 (2008).

11. J. E. Sharping, M. Fiorentino, P. Kumar, and R. S. Windeler, Opt. Lett. 27, 1675 (2002).

12. J. Lasri, P. Devgan, R. Tang, J. E. Sharping, and P. Kumar, IEEE Photon. Technol. Lett. 15, 1058 (2003).

13. D. K. Serkland and P. Kumar, Opt. Lett. 24, 92 (1999).

14. P. Devgan, J. Lasri, R. Tang, V. Grigoryan, W. Kath, and P. Kumar, Opt. Lett. 30, 528 (2005).

15. B. Cuenot, A. D. Ellis, and C. J. McKinstrie, in Procedings of the European Conference on Optical Communication (2006), paper We3.P.19.

16. T. Torounidis, M. Karlsson, and P. A. Andrekson, J. Lightwave Technol. 23, 4067 (2005). 